\title{
ÍNDICE DE CONFIANZA EMPRESARIAL - ICE
}

\section{GLOBAL}

\section{SECTORES}

Pag 7-8

\section{Resumen}

El presente artículo se realiza con la finalidad de conocer la opinión de empresarios respecto a la situación económica de las empresas de los distintos sectores del país, este estudio analiza la información obtenida por el Banco Central del Ecuador en el primer semestre del periodo 2010 - 2016. Tomando como principal referencia el Índice de Confianza Empresarial - ICE, se establece un comparativo año a año en base a tasas de variación para determinar la tendencia de este índice a lo largo del tiempo, como es el caso del sector construcción que muestra el mayor decrecimiento en el periodo de estudio.

\section{Palabras clave:}

Empresa, Confianza, Sectores Económicos y Evolución.

\section{Abstract}

This paper looks to determine the entrepreneurs opinions on the economic situation of many of the industries in a variety of economic sectors of the country. This investigation will try to analyze the information provided by Ecuador's Central Bank in the first semester of 2010 - 2016. The principal reference used is the Company Confidence Index - (ICE) and through its variation of rates one can determine the evolution of the index over time. As can be seen in the construction sector which has shown the biggest decrease during the study period.

\section{Keywords:}

Industries, Confidence, Economic Sectors, Evolution

\section{Introducción}

La información que presenta este estudio fue facilitada por la Diercción Nacional de Síntesis Macroeconómica del Banco Central de Ecuador

El Índice de Confianza Empresarial (ICE) sintetiza las expectativas de los empresarios respecto de la situación económica de su empresa y del país en general. (Banco Central del Ecuador -BCE, 2016)

El ICE se obtiene del Estudio de Opinión Empresarial (EMOE), se basa en una metodología internacional, apoyada por la Comisión Económica para América Latina y el Caribe - CEPAL. La muestra no está encaminada a una representatividad por provincia o cantón, sino se enfoca en la realización de un estudio por sectores (industria, comercio, construcción y servicios) a nivel nacional.

\section{Contenido}

Las empresas encuestadas se seleccionan mediante un muestreo dirigido por cuotas, es decir, para cada sector económico que se analiza se tiene un conjunto de unidades de observación (empresas) que son las más grandes considerando su nivel de ventas, bajo el supuesto de que estas son las que determinan la evolución de las variables que se miden dentro del estudio. (BCE, 2016)

'Economista - Analista de Investigación y Desarrollo del Observatorio Económico y Social de Tungurahua - UTA

2Economista. Magister en Gerencia Financiera Empresarial - Docente Titular Principal de Facultad de Contabilidad y Auditoria - UTA
Tabla 1. Distribución por provincia de las empresas encuestadas más representativas por sector económico en el mes de abril de 2016

\begin{tabular}{|l|r|r|r|r|r|r|}
\hline \multicolumn{1}{c}{ PROVINCIA } & \multicolumn{1}{c}{ COMERCIO } & CONSTRUCCIÓN & INDUSTRIA & \multicolumn{1}{c|}{ SERVICIOS } & $\begin{array}{c}\text { Total } \\
\text { general }\end{array}$ \\
\hline PICHINCHA & 118 & 71 & 149 & 151 & 489 \\
\hline GUAYAS & 93 & 56 & 124 & 87 & 360 \\
\hline AZUAY & 15 & 4 & 29 & 6 & 54 \\
\hline MANABI & 5 & 5 & 19 & 1 & 30 \\
\hline TUNGURAHUA & 8 & 2 & 8 & 4 & 22 \\
\hline RESTO DE PROVINCIAS & 11 & 12 & 21 & 1 & 45 \\
\hline Total general & 250 & 150 & 350 & 250 & 1000 \\
\hline & \multicolumn{5}{c|}{$\begin{array}{c}\text { Fuente: Banco Central del Ecuador - BCE } \\
\text { Elaboración: Equipo OBEST }\end{array}$} \\
\hline
\end{tabular}

En la Tabla 1, se muestra la distribución a nivel nacional por provincias y sector económico en el mes de abril de 2016, esta información fue proporcionada por el Banco Central del Ecuador, que es similar a la distribución de 2015, la misma que indica las encuestas que se aplican según los sectores y determina que el industrial aporta con 35\%; comercio y servicios con $25 \%$ cada uno y el sector construcción 15\%, además, que Pichincha es la provincia donde más encuestas se aplicó, 48,9\% respecto al total nacional, seguido de Guayas con el 36\%; Tungurahua está en el quinto puesto con el $2,2 \%$

\section{Figura 1. Evolución del Índice de Confianza Empresarial primer semestre 2010 - 2016}

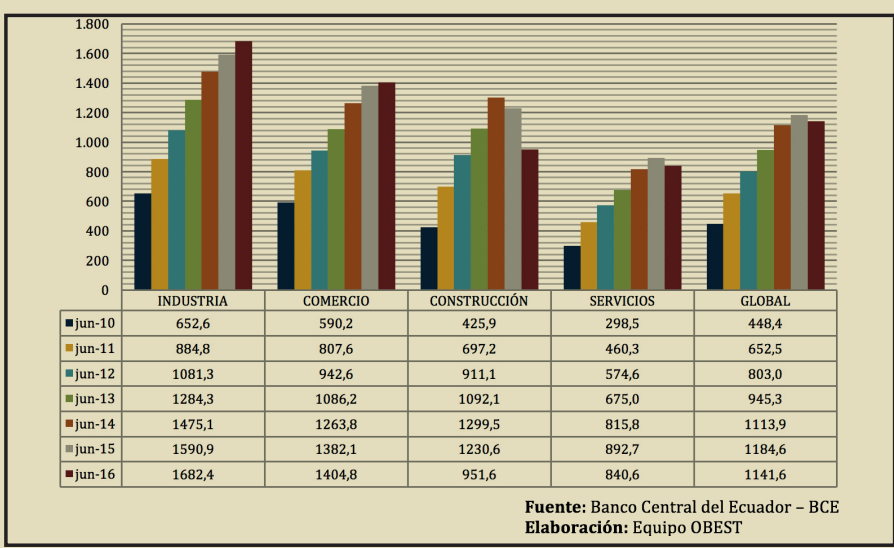

En la figura 1 se identifica la evolución del ICE por sectores en el primer semestre de 2010 al 2016. En el sector industria y comercio todos los años el índice se ha incrementado, aún cuando la tasa de crecimiento respecto al año anterior ha disminuido en 6\% y $2 \%$ respectivamente de junio 2015 a junio 2016. Para el sector construcción el ICE mantuvo un crecimiento constante hasta junio de 2014, pero disminuye en 2015 en 68,9 puntos, es decir, decrece un 5\%; en 2016, disminuye en 279 puntos, un decrecimiento de $23 \%$, siendo el indice mas bajo en este sector y, finalmente en el sector servicios el índice se incrementa hasta 2015, pero disminuye para 2016 en 52,1 puntos con un decrecimiento del 6\%; algo similar sucede con el índice global que disminuye 43 puntos, decrece $3,6 \%$. 
Figura 2. Índice de Confianza Empresarial sector Industria Enero - Julio 2016

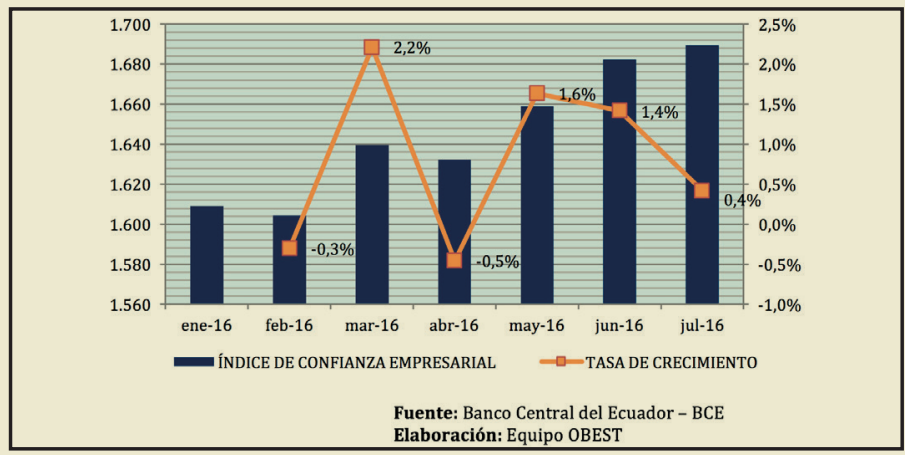

La figura 2, establece el desarrollo del ICE - Industrial durante 2016, muestra que en los meses de febrero y abril existe una disminución del índice en 4,9 y 7,5 puntos respectivamente, en cambio que con la tasa de crecimiento se observa un pico de 2,2 en el mes de marzo pero que en los últimos tres meses ha disminuido hasta cerrar en julio con una tasa de crecimiento respecto a junio de 0,4\%. La disminución en el índice puede estar dada por diversos factores que afectan a los empresarios, entre los más destacados se mencionan la iliquidez, la falta de crédito en el sector financiero, y la falta de inversión en el sector público y privado.

Figura 3. Índice de Confianza Empresarial sector Comercio Enero - Julio 2016

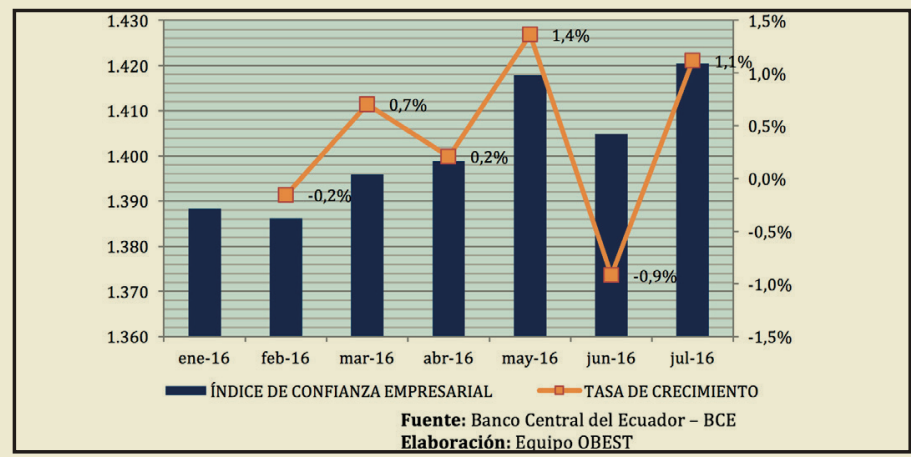

La figura 3, muestra el ICE del sector comercio, se identifica una disminución en los meses de febrero y junio donde decrecen en $0,2 \%$ y $0,9 \%$ respectivamente; y un incremento en la tasa de crecimiento en mayo y julio de $1,4 \%$ y $1,1 \%$.

\section{Figura 4. Índice de Confianza Empresarial sector Construcción Enero - Julio 2016}

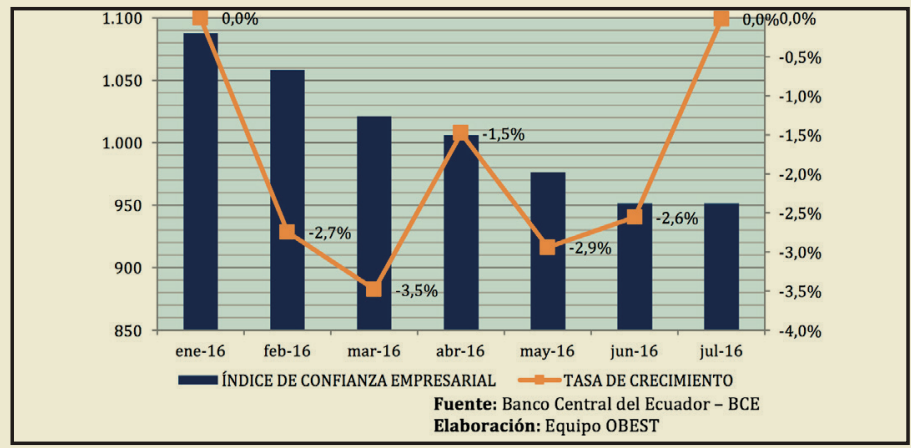

La construcción es uno de los sectores de mayor importancia dentro de la economía nacional, ya que tanto la dinámica de las empresas constructoras, como su influencia en la generación de empleo permiten señalar al sector como eje transversal para el crecimiento y desarrollo económico del país. (Mundo Constructor, 2016).
La figura 4, indica que la confianza de los empresarios del sector construcción ha disminuido progresivamente, las tasas de crecimiento durante el 2010 al 2016 registran valores negativos. El proyecto de Ley de Herencias y Plusvalía, el incremento del IVA entre otros aspectos, han provocado disminución de la demanda de casas, departamentos, oficinas, locales comerciales, lo que se ve reflejado en disminución de ventas para este sector.

\section{Figura 5. Índice de Confianza Empresarial sector Servicios Enero - Julio 2016}

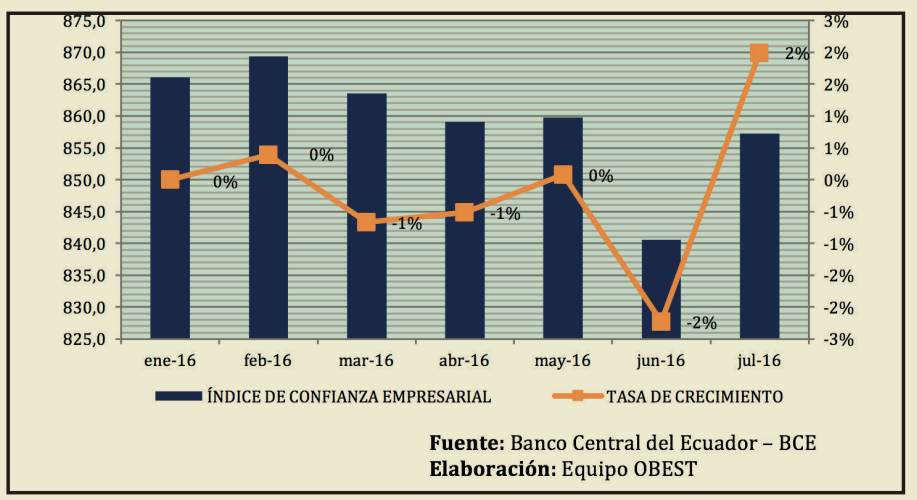

En la figura 5, el sector servicios en el período de estudio muestra un ICE que registra su mejor puntuación en el mes de febrero con 869,4 puntos; caso contrario sucede en junio, mes en donde se incrementa el IVA de $12 \%$ a $14 \%$, impactando de manera directa a este y los demás sectores objeto del presente estudio.

Figura 6. Índice de Confianza Empresarial Global Enero - Julio 2016

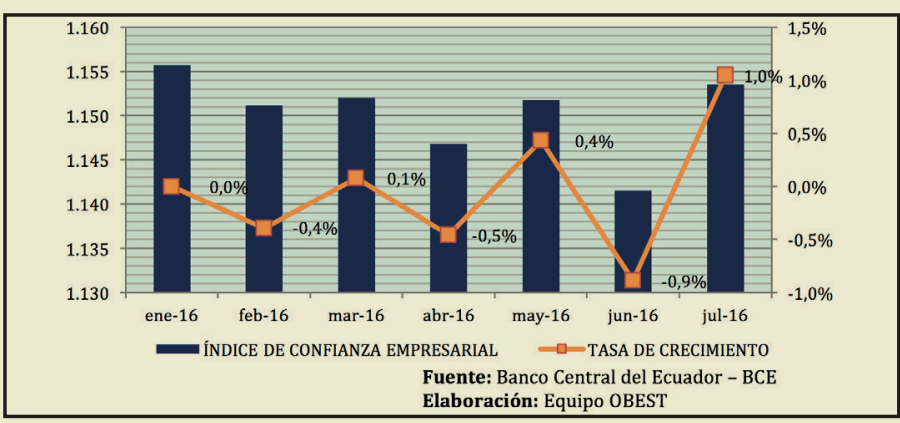

El índice de confianza empresarial global se obtiene ponderando Ios ICE sectoriales y por tanto, también estos ponderadores afectan a la contribución de cada sector al ICE Global mensual. (BCE, 2016)

Finalmente, el índice de Confianza Global refleja en julio un incremento de 11,9 puntos, es decir, creció en 1\% en relación a junio, mes en el que se registra el menor índice en lo que va del año con $1.141,6$ puntos; con un decrecimiento del $0,9 \%$.

\section{REFERENCIAS}

-Banco Central del Ecuador - BCE, disponible en: https://www. bce.fin.ec/index.php/component/k2/item/764-indicadores-de-coyuntura

-Banco Central del Ecuador - BCE, disponible en: https://www. bce.fin.ec/index.php/component/k2/item/314-estudio-mensual-de-opini\%C3\%B3n-empresarial

- Jaramillo, A. (2016, 20 de abril). El sector de la construcción en un difícil 2016, Mundo Constructor. Disponible en: http://www. mundoconstructor.com.ec/construccion/comercial/544-el-sector-de-la-construcci\%C3\%B3n-en-un-dif\%C3\%ADcil-2016.html 